Meta

Journal des traducteurs

Translators' Journal

\title{
Entretien sur la traduction avec Edgar Morin
}

\section{Alexis Nouss}

Volume 40, numéro 3, septembre 1995

La traduction, qu'est-ce à dire? Phénoménologies de la traduction

URI : https://id.erudit.org/iderudit/003661ar

DOI : https://doi.org/10.7202/003661ar

Aller au sommaire du numéro

Éditeur(s)

Les Presses de l'Université de Montréal

ISSN

0026-0452 (imprimé)

1492-1421 (numérique)

Découvrir la revue

Citer cet article

Nouss, A. (1995). Entretien sur la traduction avec Edgar Morin. Meta, 40(3), 343-351. https://doi.org/10.7202/003661ar

\section{Résumé de l'article}

Penser la complexité est une des visées majeures de l'oeuvre et de la réflexion d'Edgar Morin. au carrefour des sciences physiques, biologiques et humaines. À cette fin, il tente de dégager les niveaux et les systèmes d'organisation du réel ainsi que de la connaissance du réel. Épistémologue autant que sociologue, l'auteur de La méthode (Seuil, 1973, 1977, 1980) est un infatigable poseur de ponts interdisciplinaires. Analysant ainsi le principe du savoir dans un monde mû par un "désordre organisateur", régi par un principe d'incertitude et un jeu général d'interactions, il examine au cours de cet entretien la place de la traduction dans sa pensée et aussi dans son itinéraire personnel. 


\title{
ENTRETIEN SUR LA TRADUCTION AVEC EDGAR MORIN
}

\begin{abstract}
One of the primary objectives of the work of Edgar Morin - a theorist at the crossroads of the physical biological and social sciences - is to reflect on the notion of complexity. He seeks io reveal the systems and levels of both the organization and the knowiledge of reality. An epistemologist and a sociologist, the author of La méthode (Seuil, 197.3. 1977. 1980) spares no effort in his quest to build bridges betw'een disciplines. Analyzing the principles of knowledge in a world fuelled by "organizational chaos," and subjected to uncertain. $y$ and the general rules of interaction. the author shares his thoughts on translation and discusses its place in his ow'n intellectual and personal pursuits.

Résumé

Penser la complexité est une des visées majeures de l'auvre el de la réflexion d'Edgar Morin. au carrefour des sciences physiques, biologiques et humaines. À cette fin, il tente de dégager les niveaux et les systèmes d'organisation du réel ainsi que de la connaissance du réel. Épistémologue autant que sociologue, l'auteur de La méthode (Seuil. 1973, 1977. 1980) est un infatigable poseur de ponts interdisciplinaires. Analysant ainsi te principe du

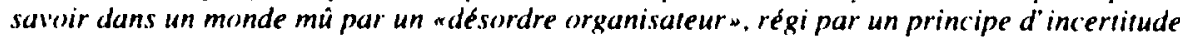
et un jeu général d'interactions. il examine au cours de cet entresien la place de la traduction dans sa pensée et aussi dans son itinéraire personnel.
\end{abstract}

Alexis Nouss : Toute votre auvre cherche détablir des passages entre differents champs du savoir. De l' un d l'autre. est-ce que ca communique ou est-ce que sa se traduit?

Edgar Morin : Je ferai une remarque introductive ou préliminaire : pour moi, et c'est la thèse que j'ai développée dans mon livre La connaissance de la connaissance, toute connaissance est traduction et en même temps construction ou reconstruction. C'est une traduction d'un type très intéressant. Dans la vision. par exemple, il y a des stimuli qui frappent les cellules diverses de ma rétine et ceci, à partir de ce moment-là, est transformé par le nerf optique dans un code binaire, donc traduit, mais cette traduction codée est elle-même travaillée et retravaillée dans diverses régions du cerveau et nous avons alors une vision qui, évidemment, n'est pas un reflet des choses mais une traduction. Je trouve donc que, paradigmatiquement, tout ce qui est cognitif est traduction. La traduction qu'est notre perception, c'est une traduction à partir du silence et du bruit à la fois : le bruit, c'est-à-dire toute une série de stimuli en désordre que nous ordonnons. auxquels nous donnons un sens. Dans quelle mesure est-elle fidèle ? Nous ne le savons pas. Nous savons qu'il y a des cas où elle n'est pas fiable : quand il y a une hallucination. nous croyons voir quelque chose. En réalité. cette vision se produit uniquement dans notre esprit. Nous avons alors des moyens de vérifier la fiabilité de la traduction de notre vision en nous concertant avec autrui. En nous concertant et en réfléchissant, nous faisons la différence entre ce que nous avons vécu dans le rêve et ce que nous avons vécu à l'état de veille. Finalement, nous utilisons différents moyens pour vérifier la fiabilité mais la fiabilité n'est pas la fidélité, nous savons qu'il y a des ultrasons qui nous échappent. Nous arrivons donc à cette idée : nous vivons, nous pensons, nous voyons à partir de traductions.

A.N. : La traduction serait donc déjd un fait hiologique, inscrit dans le corps?

E.M. : La perception est une traduction. L'intéressant est que notre cerveau qui fixe tous ces processus de traduction, donc de perception, dont nous sommes inconscients, est enfermé dans notre boîte crânienne, il ne communique pas directement avec le monde 
extérieur, il communique par l'intermédiaire de nos appendices sensoriels el je pense qu'il y a un rapport dans toute connaissance qui est à la fois fermeture et ouverture, séparation et inhérence : dans l'immédiateté. il n'y a pas de connaissance possible. L'autoconnaissance de soi - Montaigne ou autre - nécessite une certaine distanciation de soi par l'esprit d'objectivation ainsi que d'autres examens de soi : il faut se détacher de soi. Du reste, je cite souvent cet apologue de Spencer Brown, le mathématicien anglais, qui disait : «Si le cosmos voulait se connaître, il serait obligé de dégager hors de lui un pédoncule qui s'éloignerait suffisamment, puis il faudrait qu au bout du pédoncule il y ait une capacité cognitive, c'est-à-dire quelque chose d'original qui se développerait, et au moment où cette extrémité du pédoncule connaissant la capacité cognitive sé retourne dans le cosmos, cette réussite est en même temps un échec puisque le pédoncule est trop séparé tout en faisant partie du cosmos". C'est exactement le cas de l'être humain : nous sommes issus du cosmos et par le cerveau qui est quelque chose d extrêmement original, nous connaissons ce cosmos et nous payons justement cette connaissance par la séparation. Pour moi c'est à ce niveau-là. je dirais paradigmatique, je dirais biologique et, bien entendu, anthropologique, que j'utilise le terme de traduction.

En ce qui concerne votre question sur le caractère transdisciplinaire, je crois qu'il faut le voir à deux niveaux : d'un côté, je crois qu'il y a des concepts organisateurs qui traversent les disciplines en prenant des formes différentes dans chacune, et c'est cela qui rend possible la communication. Par exemple, il y a le concept d'organisation, qui concerne les systèmes. Il est certain que nous avons des systèmes : le noyau d'un atome est un système, l'atome est un système, un astre est un système. les molécules sont des systèmes. les êtres sont des systèmes, les sociétés aussi, etc. Ce mot ceuvre partout et l'on n'est pas plus avancés. Ce qui est intéressant. c'est de savoir qu'un système est quelque chose de plus et de moins que la somme des parties qui le constituent, c'est de faire une théorie de l'organisation qui permet de rayonner dans les différents secteurs. Mais là encore, cela ne suffit pas. Par exemple. dans ce que j appelle l'auto-organisation, mieux l'auto-éco-organisation, qui est un phénomène où l'être s'auto-produit, s'auto-organise dans une relation singulière avec son environnement où il puise de l'énergie, de l'information, etc., nous avons aussi un autre niveau de l'idée de système et si je veux utiliser ce niveau pour comprendre la société, je le ferais différemment que pour comprendre un organisme vivant.

À mon avis, il faut donc un certain nombre de concepts, de paradigmes de base pour pouvoir faire communiquer les disciplines. Ainsi, comprendre que dans notre univers il y a toujours une combinaison, un cocktail incertain et différent d'ordre. de désordre el d'organisation, cela vaut pour le monde physique à sa façon puisqu'il commence par une sonte de rayonnement désordonné, mais ça vaut pour la société humaine qui fonctionne avec des désordres qu'on appelle les libertés, f̧a vaut pour l'écologie, pour la biosphère, etc. Autrement dit, sans certains principes fondamentaux qui sont en rupture avec le déterminisme mécanique, avec le réductionnisme, avec l'incapacité de comprendre la complexité - comprendre l'autonomie par exemple, et l'individualité, et la conscience - il est impossible de faire la moindre communication. Je dirai donc qu avant de songer à la traduction, il faut avoir - et c'est ce que j’appelle le mode de pensée complexe - certains principes : le principe dialogique ou le principe hologrammatique qui dit que non seulement la partie est dans un tout mais que le tout se trouve aussi dans la partie, le principe de la récursion de la boucle... Ces principes, selon l'objet. se manifestent chaque fois d'une façon spécifique originale. Il ne suffit pas d'avoir la connaissance de ces principes pour pouvoir les appliquer automatiquement à toutes les disciplines. On est obligés de passer par le concret des différences, e'est une chose fondamentale. 
Par ailleurs, il y a des concepts baladeurs, et toute l'histoire des sciences en a été fécondée. Prenez la notion de travail, une notion qui relève évidemment de l'expérience sociale : le travail est ce qui est réservé à l'esclave ; cette notion a pu être extirpée de l'expérience sociale, comme la plupart des notions physiques, on connaît les origines de la géométrie, de l'arithmétique. Prenez donc le travail et l'idée de force et quand vous unissez les deux, est créé le concept d'énergie. Puis ce concept d'énergie qui a un sens physique, qui peut être mesuré, qui peut être reconnu sous ses différentes formes, et dont la forme calorifique va introduire au principe d'entropie, ce concept d'énergie peut circuler un peu partout sous des formes originales. Prenez le mot d'information. voici aussi une notion qui a pris un sens mathématique dans la théorie de Shanon et qui est revenue sur la biologie génétique puisqu'on a dit que les gènes contiennent de l'information. Vous avez des notions qui sont comme des virus, et les virus, ou bien ils sont très méchants, ils détruisent tout, ou bien, au contraire, ils s'introduisent jusque dans le génome et ils apportent une propriété nouvelle. Mandelbrot, je crois, disait que le progrès des sciences se fait souvent par des tranports d'une idée d'un domaine à un autre, faits par un chercheur talentueux ou génial.

Tous ces termes-là se trouvent retraduits dans chaque champ de compétence. II y a un jeu de circulation mais le concept qui circule n'est pas le même à l'arrivée : par exemple. dans le monde des sciences humaines et dans le monde des sciences physiques, et vice versa. Il y a un jeu incessant qui au départ se fait toujours de façon contrebandière : on veut enfermer les concepts dans des disciplines mais parmi les concepts, beaucoup sont nomades.

A.N. : Dans cette perspective. la traduction est-elle simplement la dynamique de passage de ces principes haladeurs ou est-elle un principe organisateur?"

E.M. : La traduction fait partie. bien nécessairement, de l'implantation de tel concept venu d'ailleurs dans un système théorique où il n'a plus le même sens que celui qu'il avait pris au départ: souvent, elle se fait selon un processus inconscient ou très peu conscient. Il existe un grand obstacle dans l'organisation disciplinaire qui est une organisation arbitraire du savoir puisque, dans le fond, on fail entrer des objets, découpés sur la discipline, qui en général ne sont pas des objets naturels : il y a une abstraction et une extraction. Les disciplines ne peuvent pas operer entre elles de traduction mais, par contre, quand vous avez une possibilité de concepts organisationnels, vous pouvez. effectuer des traductions.

J'ajoute qu'il y a des cas où nous butons sur une non-possibilité de traduction. définitivement ou non. Vous étudiez. par exemple le cerveau et l'esprit. Si vous les étudiez sous un angle bio-électrico-chimique, vous étudiez. une machine formidable qui communique à travers des interactions intemeuronales et vous pouve $z$ décrire des processus avec des choses tout à fait spécifiques qui sont biologiques - les synapses, les neurones - ou bien électrico-chimiques. Vous avez donc un langage pour parler de ces choses-là mais ces choses-là ont des correspondances en même temps sur le plan de l'esprit - mind. mente. le français n’a pas de mot pour dire le mental. Ces phénomènes se manifestent sous forme de paroles, d'idées, de discours. Il est évident que l'on ne peut pas traduire d'un niveau a l'autre. Nous sommes obligés de faire coexister les deux langages et de nous rendre compte qu'il y a des conséquences quand une perturbation sur un plan opère sur l'autre. Vous avez une poussée d'adrénaline qui va même modifier vos idées mais par ailleurs vous avez une émotion qui vient de votre esprit et qui va elle-même modifier la machine cérébrale. C'est la même chose. l'esprit et le cerveau, mais en même temps vous les prenez sur deux angles : pourquoi ? Parce que tout ce qui est mental est émergence, et tout ce qui est émergence est par principe indéductible et non réductible. Les propriétés 
de la vie qui sont l'auto-reproduction. l'auto-organisation, la connaissance d'un milieu, etc., n'existent absolument pas au niveau de chaque molécule isolément, ce sont des propriétés de l'organisation complexe. II a fallu l'hypercomplexification humaine pour que les processus prennent forme à travers le langage, forme de mental et forme de conscience. Ce sont des émergences, donc vous ne pourrez jamais les traduire en termes cérébraux : toute tentative de faire ce type de traduction réductrice est vouéc à l'échec. Vous ne pouvez pas non plus l'abstraire et faire comme si le cerveau n'était qu'un transmetteur de télévision qui sert de relais à des messages d un esprit intemporel.

A.N. : Peut-on - ou doit-on - lier la notion de traduction et le principe d'incertitude sur lequel wous avez travesillé? Là on la certitude assurerait une communication directe, le principe d'incertitude obligerait à une traduction incessante d'un phénomine à un autre.

E.M. : Absolument. D’abord, l'incertitude n'est pas absolue. Nous naviguons dans un océan d'incertitude mais à travers des archipels de certitude. Nous avons toujours des certitudes qui sont locales, qui sont situées et datées, qui sont particulières. Nous n'avons pas de certitudes fondamentales parce que effectivement, il n'existe aucun fondement de certitude ni à la science ni à la philosophie, mais ça ne rend pas la science et la philosophie impuissantes el hégayantes: la preuve en est que la connaissance scientifique s’accroît et que la philosophic pxut continuer à réfléchir justement sur son absence de fondements. Nous sommes un peu comme le baron de Münchhausen - c'est le problème de l'auto-organisation : par les lacets de nos chaussures, nous nous élevons dans les airs: par un circuit rotatif, nous pouvons nous élever sans avoir de fondements. D'ailleurs, tout le vice du mode de pensée architectural était le besoin d'avoir une pierre fondamentale comme pour une maison : mais si vous avez un mode de pensée qui est musical, il est évident que le mouvement thématique et symphonique entraînera la chose. Dès que l'on est dans l'incertitude, on ne peut que parier. La stratégie cognitive consiste à essayer de capter le maximum d'informations, de s'appuyer sur le maximum de certitudes locales et partielles pour s'avancer dans l'incertitude et faire un certain nombre de paris qui vont $s^{\prime a}$ avérer les uns féconds, les autres nocifs. Bien entendu, au cours de ces processus, nous ne faisons que traduire puisque la connaissance elle-même est une traduction permanente. Pour en revenir à celle idée d'information. il n'y a pas que la notion de traduction. Nous extrayons ou plutôt nous arrivons à voir à partir du bruit ou du vide quelque chose qui nous résout une incertitude: Tristan arrive avec une voile blanche, fa veut dire «tout va bien", mais malheureusement il peut y avoir une erreur, un mensonge qui va vicier l'information. Tout signal qui veut dire, par exemple, "Waterloo a été gagné par les Anglais», porte une certitude et résoul une incertitude. L'information est quelque chose, si elle est vérifiée. qui va nous faire sortir de l'incertitude. Après vient un autre problème : quel est le sens de la défaite de Waterloo? Quelles vont être les conséquences pour la France, pour l'Europe, etc. "? Nous faisons des hypothèses...

A.N. : Nous faisons alors de la traduction au sens herméneutique.

E.M. : Tout à fait. parce que toute activité cognitive n'est pas seulement faire de la traduction de façon brute, elle consiste à faire une traduction qui vous permet de situer. c'est-à-dire de contextualiser et éventuellement de globaliser. On vous dit : aOn bombarde Sarajevo». Pourquoi? Et pourquoi les Serbes ? Si vous avez un certain nombre de connaissances historiques, géographiques, etc., vous comprenez parce que vous avez contextualisé pas seulement géographiquement mais aussi historiquement. Et si vous pouvez. globaliser dans les Balkans. c'est encore mieux. L'interprétation d’un événement se fait en mobilisant un ensemble de connaissances, qui va nous permettre de lui donner un sens. interprétation-traduction fonctionne toujours. 


\section{A.N. : Information et traduction coexistent donc dans ce processus?}

E.M. : Pour s'en refferer à la forme sommaire de la théorie de l'information, vous avez un émetteur, vous avez un récepteur ; bien entendu, ils ne peuvent communiquer que parce qu'ils ont un code commun, un langage commun, le français par exemple. Ils communiquent : l'un transmet une information a l'autre qui comprend parce qu'il a tout de suite * le code commun. S'il n'a pas le code commun, un type parle anglais et l'autre français, le français va faire appel à un traducteur. L'information se situe toujours dans un cadre où les gens sont capables de communiquer l'un avec l'autre. Bien entendu, le propre de la communication qui vaut pour toute communication, y compris pour la traduction d'un livre d'une langue à l'autre, c'est le bruit - noise —., c'est-à-dire que l'intégralité n'est pas entièrement conservée. Il faut lutter contre le bruit : comment traduire?

Si on parle de la littérature, on entre dans le domaine de la complexité. Un roman. et plus encore un poème, par un écrivain, c'est quelqu'un qui parle dans une langue et qui, parlant dans une langue, crée sa propre langue au sein de cette langue. II la crée, même pas de façon volontaire, il est comme ça, il crée son univers. Le traducteur va essayer de traduire ça dans une autre langue et il va donc se trouver devant un double impératif. Ou bien il va être fidèle littéralement, avec une grande déperdition, une grande difficulté parce que les mots ne coïncident pas exactement : il existe des trous dans chaque langue et ce trou qui existe dans une langue est rempli par un mot qui existe dans une autre langue ; la fidélité absolue n'est pas possible. Ou bien, il va essayer de donner un analogon, de reconstituer par d'autres moyens l'univers, et alors, il risque aussi d'être infidèle. Un poème, par exemple, il va essayer de le traduire en vers français bien que la versification soit autre, il peut faire quelque chose de joli, mais ce ne sera pas la même chose : ou bien il va faire une traduction littérale, et à ce moment-là, toute la musique, tout ce qui est connotation, va disparaître.

En poésie. c'est terrible parce que tout tourne autour de la connotation, du jeu des mots, etc. En littérature, ça dépend. J'ai lu Dostö̈evski à partir de 14, 15, 16 ans, je l'ai lu dans les premières traductions, celles de Melchior de Vogué qui étaient des traductions francisantes, qui essayaient d'enlever tout le langage râpeux de Dostoïevski. De plus il éliminait des passages qui, pensait-il, pouvaient ennuyer le lecteur français, il voulait rendre Dostoïevski comestible. Aujourd hui, après toute la succession de traductions, les ultimes étant de Markowicz, on se rend compte de tout ce qui a été enlevé. Il n'empéche que pour moi, j'ai reçu le choc, avec violence, parce que de Vogúé n'a pas pu faire que l'univers de Dostoïevski, l'être de ces personnages des Freres Karamazov, des Possédés, ne s'impose à moi avec une force telle en dépit de la mauvaise traduction. Pour moi, l'essentiel du message est passé et après, quand je lis les demières traductions, celles de Markowicz - il a traduit Le joueur, il a traduit Les nuits blanches - je trouve un plaisir extraordinaire mais je me dis que ces cuvres m'avaient déjà beaucoup frappé dans leur traduction imparfaite. Je prends un autre exemple : Hegel, La logique... Hegel a eu une première série de traductions en France vers 1860 , un type qui s'appelait Vera. d'origine italienne et qui s'était dit : “Je vais rendre ça concevable pour les Français parce qu'ils ne vont rien comprendre de ces trucs". Tous les hégeliens disent que c'est une traduction absolument abominable. Par ailleurs, j'ai lu La phénoménologie de l'esprit dans une traduction réputée bonne, qui était celle d'Hyppolite. Eh bien pour moi, ce livre que j'ai donc lu à vingt ans $\mathrm{m}$ 'a fait un effet extrêmement fort et $\mathrm{j}$ 'ai l'impression que tout ce qui avait été appauvri était secondaire. De même Marx, Le manuscrit économicophilosophique de Marx, tout le monde $\mathrm{m}$ 'a dit que cette traduction dans laquelle je l'ai lu - dans ses Euvres complètes des années 1930 - était absolument ignoble mais il n'empêche que le choc des idées fondamentales était là : je les ai prises, assimilées, et puis après, quand $\mathrm{j}$ 'ai lu la bonne traduction, j'ai dit «oui, en effet. c'est bien mieux, mais 
enfin...». Il ne faut pas trop se biler: quand un ouvrage est nouveau, une traduction, même mauvaise, est bonne. Parce que quelque chose de la complexité originelle passe. il faut vraiment qu'il y ait un sabotage incroyable pour que ça ne passe pas. Autrement dit. la force d'une pensée. d'une cuvre. passe à travers les traductions même médiocres.

A.N. : Est-ce une question d' historicité. de partage de la même historicité par le traducteur et le lecteur?

E.M. : Entendons-nous, la traduction de Vera, c'est de 1860). Moi, je le lis en 1942. De Vogüé, il traduit à la fin du XIX $X^{c}$ siècle. La question de l'historicité est intéressante parce qu'on a besoin à chaque époque de refaire une traduction, que ce soit de Shakespeare, de Cervantès. Pourquoi ? C'est notre langue qui a changé. Comme les langues vivent, au cours de leur vie elles incorporent des mots nouveaux, elles oublient des mots anciens. Elles ont une façon de rendre compte des choses qui s'est modifiée : c'est une véritable régénération de l'ceuvre qui se fait à travers les différentes traductions. D'ailleurs, on se rend bien compte qu'il y a des traductions classiques, des traductions romantiques et des traductions contemporaines. Je pense que la traduction est un travail inachevé et inachevable et que l'état nouveau d'une langue appelle une traduction nouvelle. Les Mille et une Nuits, vous avez la traduction classique de Galland qui était une mutilation ; après vous avez eu la traduction de Mardrus au début du siècle. On voit très bien que ce n'est pas seulement l'état vivant de la langue mais aussi des conditions culturelles qui appellent le renouvellement de la traduction.

A.N. : La fonction de la traduction est-elle d'effacer la distance entre les langues et les cultures ou, au contraire, de la signifier?

E.M. : La traduction est confrontée à un double impératif contraire : elle doit faire les deux. Les traductions de Markowicz de Dostö̈evski rendent le caractère râpeux qui est celui de l'écriture de Dostöevski et qui jusqu’à présent était un peu gommé : on sent que c'est dans une autre langue mais, en même temps. c'est du français. La poésie, elle. appelle l'édition bilingue, très utile quand on est dans le même type de langues parce que si on ne connaît pas les caractères arabes ou les caractères grecs ou les caractères chinois. par exemple, le bilingue ne sert à rien. Cependant, celui qui connaît un petit peu une langue, moi, par exemple - pour les poètes espagnols surtout ou pour l'allemand mais je le connais très mal - je veux avoir ma traduction bilingue: je peux alors vraiment goûter le poème, parce que je me plonge dans l'une et l'autre langue. Grâce à la traduction. je peux pénétrer dans les mots allemands que je ne connaissais pas auparavant. et grâce à ces mots allemands je peux donner une résonance à la traduction qu elle n'aurait pas sinon.

A.N. : La traduction de la poésie, ou de la littérature en général, demande-telle un talent particulier?

F.M. : Dès qu'il s'agit de littérature ou de poésie. il y a un problème spécifique qui n'existe pas quand vous assister à un colloque scientifique où vous mettez lés écouteurs et où ça n'a pas d'importance, à moins que l'orateur ait un talent particulier et que le traducteur simultané, s'il n'est pas génial, fasse perdre l'essentiel des choses. En fait. il y a deux arts, celui du traducteur simultané du colloque qui doit être dans l'immédiat. toujours globaliser très vite, et puis l'art du traducteur littéraire qui. lui. a le temps. Il regarde, il médite, il réfléchit : ce n'est pas seulement une langue dans l'autre mais un univers global qui doit être traduit dans l'autre langue et ça, c'est l'art. L'art, à mon avis. est toujours la solution de deux impératifs contraires. C'est toujours quelque chose d'impossible avant et dont on se rend compte que c'est possible après. Je sais qu'il y a des époques où des gens disent : "Bon, moi, je vais être fidèle à la lettre principalement”. 
et d'autres où on dit : «Bon. je vais essayer d'être fidèle soit au sens, soit à la musique», etc. Bien entendu, l'idéal ce serait d'avoir les deux a la fois mais l'un tend à exclure lautre. À mon avis, la traduction doit essayer de jouer sur les deux tableaux même si elle ne peut pas à la fois réussir à jouer sur l'un et l'autre. Par exemple, je prends un vers de Machado que je cite tout le temps mais que je traduis au fil des ans différemment: "Caminante no hay camino, el camino se hace al andar».

"Cuminante": «toi le cheminant". Il n'y a pas de mot en français pour le dire : «cheminot", ce n'est pas le mot. Cheminant, marcheur, oui, peut-être. Au début, je disais «toi qui chemines", mais ce n'est pas ça. "No hay camino», «il n'y a pas de chemin». Cela, ça va. Mais : «el camino se hace al andar», le chemin se fait «al andar». Il se fait en marchant. ce n'est pas ça. Il se fait en allant, "al andar», dans le fait d'aller. Donc je modifie ma traduction et du point de vue «caminante», le premier mot et du point de vue «al andar», le dernier mot, je n'ai absolument pas la possibilité de traduire. Et je sais que la périphrase alourdit, «toi qui chemines», ca prend une interjection et je sais qu'elle est implicite, puisque c'est un appel. Je me rends compte qu'à chaque fois, selon l'humeur, je fais une traduction différente.

A.N. : Si la traduction est cet acte humaniste qui consiste d aller vers l'autre, ne perdelle pas sa fonction aujourd' hui, dans cet univers que les technologies, notamment celles de l'information, ont uniformisé, un univers de la transparence, en somme, et donc un univers de la non-traduction?

E.M. : En réalité, les deux univers se superposent. Vous avez un univers de l'homogénéisation culturelle, qui a sa langue et qui n'est pas l'anglais, qui est une sorte de basic english, un anglais véhiculaire pauvre qui n'a rien à voir avec l'anglais de la littérature. Vous avez cet univers-là qui fonctionne avec les MacDonald, mais vous avez quand même l'autre univers, les bistros, la gastronomie régionale et des retours vers cette gastronomie. Regardez du point de vue de l'édition, il est quand même frappant qu'en France, on traduisait avant tout les romans anglais, les romans allemands, au début du siècle, et que maintenant on traduit des romans de toutes les langues, y compris des romans de petites langues, Ivo Andrić. des romans serbes, etc. Regardez tout ce qu'il y a de traduit en japonais qui n'existait pas. Non, je pense que les deux univers sont des univers ennemis mais dont l'un et l'autre continuent leur propre développement, leur propre devenir.

A.N.: Puisque vous évoquez les petites langues et pour aborder un theme plus personnel. vous qui venez d' un milieu où l'on parlait d'autres langues (et je pense au tres beau livre que vous avez consacré d votre père), la vôtre, le français, n'est-elle pas, d'une certaine maniere, une langue de traduction?

E.M. : Dans mon cas c'est difficile parce que je suis né à Paris, ma famille parlait le français, mais enfant, j'entendais parler l'espagnol, le vieil espagnol, entre mes parents et leurs parents ; entre eux ils parlaient français mais il arrivait qu'ils parlent espagnol, entraînés dans une conversation spécifique. Cet espagnol que $j$ 'ai entendu pendant toute mon enfance, pas toujours, pas constamment, je l'avais dans les oreilles puis il a dormi ; mais jamais je n'en ai prononcé un mot. Or, je vais en Espagne et je suis très étonné de voir qu'il y a beaucoup de choses que je comprends et il faut très peu de temps pour faire des accommodations : par exemple. dans mon espagnol familier il n'y avait pas la jota : on disait par exemple fijo pour dire fils au lieu de dire hijo. Les accommodations $m$ 'ont été très faciles et assez rapidement, sans avoir appris l'espagnol, allant souvent en Amérique latine, je suis parvenu à faire des conférences, des cours. dans un charabia hispanique. Quant à l'italien... Certes ma famille était originaire de Livoume, ma grandmère matemelle avait encore quelques proverbes italiens qu'elle aimait réciter mais 
j'avoue que cette langue, je ne l'ai jamais connue. Cependant étant en Italie. j'ai aimé l'Italie, j'ai aimé l'italien et là aussi en quelques années, par amour pour l'italien, je me suis mis à faire aussi des exposés, et quand je parle à des gens, j'essaie de parler italien. Là. maintenant, je suis en train de lire un livre en italien, le livre de Cavallari qui s'appelle La fuga de Tolstöi, le récit très minutieux de la fugue de Tolstoï. II y a des mots qui me manquent, mais je suis épris de cette langue.

En ce qui me concerne donc, je ne suis pas parti d'une langue première, d'enfance par exemple, sur laquelle le français est venu comme langue seconde. C'est même le contraire : l'espagnol était une langue périphérique.

A.N.: Vous venez néanmoins demployer un signifiant superbé : l'espagnol dormait en vous. Ça évoque tout de suite pour moi Kafka qui rêvait d'une langue perdue ou oubliée. le yiddish pour lui, comme l' hébréu pour d' autres...

E.M. : Dans l'itinéraire de ma famille, je me rends compte qu'il y a tout un passage par Livourne, et donc l'italien, qu'ils ont perdu ou presque perdu à Salonique. Il y avait encore des mots italiens chez ma grand-mère, je vous le disais, mais le passage par l'Italie a été oublié. Il est revenu, mais il faudrait peut-être alors aller dans un inconscient encore plus profond. C'était le grand-duché de Toscane, c'était donc la Toscane qui était ma fascination.

Et quant à cet espagnol ancien, antique. c'est vrai, il dormait. il s'était déposé en moi, il s'était trouvé inactivé. Je n'ai jamais éprouvé le besoin de le parler parce qu'avec mes grands-mères je parlais le français, elles me comprenaient parfaitement. Je n’ai jamais eu la nécessité de parler cet espagnol. et c'est ce qui $\mathrm{m}^{\prime}$ a frappé : je ne l'ai jamais parlé mais it m’était familier, plus que familier: il m'était familial. J'ai découver l'espagnol avec de l'amour pour l'Espagne puisque cela était en moi. Je vis en Europe en ayant le sentiment qu 'incontestablement la France est ma patrie mais que j'ai une patrie méditerranéenne avec des points forts qui sont l'Espagne et l'Italie.

Et pour les mots, les Balkans aussi : cet espagnol-là avait subi l'influence ottomane. Il y avait quelques mots turcs et notamment deux mots qui sont capitaux et qui sont : aïdé et yavash. Aïdé. c'est un mot impératif qui veut dire : “allons, allons». c'est aussi bien le mot que dit le père pour éveiller son fils que le chef de chantier pour faire travailler ses ouvriers, c'est le mot du commandement, du dynamisme. Yavash, c'est le mot contraire. C'est langsam comme dans Wozzeck, c'est : "Vas-y mollo", «doucement», etc. On a tout le temps: «Ne nous pressons pas»... Alors avec aïdé et yarash vous contrôlez presque tout l'univers des comportements. Donc, je suis en Macédoine ex-yougoslave. Et quelle est ma surprise, mon émerveillement, d'entendre ä̉dé et yavash. Aussitôt, une familiarité profonde s'est réveillée, balkanique, et de plus, évidemment, un certain nombre de nourritures que j’ai reconnues. Il existe un complexe dans lequel intervient aussi la nourriture avec ses mots propres. Même quand on parlait français, le gratin d'aubergines, on l'appelait le sfongadico. Pour moi. c'est le sfongadico. Il y a donc quelques mots pour moi qui relèvent de ces nourritures matricielles, qui existent dans cette langue el qui $n{ }^{\circ}$ ont pas de mots français équivalents. Je peux dire «le gratin d'aubergines», mais le sfongadico n'a rien à voir avec le gratin qu'on peut vous servir ailleurs.

A.N. : La multiplicité des languess proure-t-elle que le principe de complexité que vous développez est le seul qui nous permette d' aller à la rencontre du monde?

E.M. : Incontestablement. Seulement, il faut voir qu'il y a l'un et le multiple puisque toutes ces langues ont les mêmes principes structuraux d'organisation, détectés par la linguistique structurale : et cependant, avec ces principes fondamentaux, une diversité absolument extraordinaire et les langues qui ne demandent qu'à se diversifier et se multiplier. Le problème de la complexité. comme pour les cultures, c'est l'unitas multiplex, 
l'unite dans la multiplicite. De méme, nous avons des choses fondamentalement anthropologiques comme le rire, le sourire ou les pleurs, qui sont modulés, utilisés, reprimés ou exhibés de façon tout a fait différente selon l'éducation, selon les cultures. Ce qui veut dire que méme les langues les plus étrangères peuvent etre traduites. Autrement dit, pour qu'il y ait traduction, c'est-à-dire pour qu'il y ait connaissance, il faut qu'il y ait à la fois séparation et inhérence. L'inhérence, c'est qu'effectivement nous avons une structure commune. Du fait de notre inherence au monde animal aussi, quiconque a un chat, un chien, peut très bien communiquer avec lui. Mais nous ne pourrions pas communiquer avec des êtres qui seraient dans un monde obéissant à des lois physicochimiques radicalement différentes des nôtres.

A.N. : La traduction comme cause première de la communication?

E.M. : Pour communiquer, il faut être séparés, il faut avoir aussi un principe commun. Vous avez le complexe connaissance-communication-traduction, etc. Tout ça renvoie l'un a l'autre, il n'y a aucun terme privilégié, il faut un macro-concept qui unisse toutes ces notions pour comprendre ce dont il s'agit.

A.N. : Ce macro-concept, vous ne le nommez pas?

E.M. : Non. Si je parle de la connaissance, je dis que la connaissance suppose la traduction, la reconstruction, l'inhérence. la séparation. Et si je veux parler de traduction, je dirais que la traduction va se référer à un code, etc. Selon la chose sur laquelle je focalise, c'est comme une constellation qui se dispose différemment.

A.N. : Vous traduisez ce macro-concept selon le systeme qui doit l'accueillir.

E.M. : Je modifie la configuration des termes.

A.N. : Si certains parlent de l'impossibilité de la traduction, elle demeure pour vous un concept positif.

E.M. : Dans toute traduction il y a une perte. Mais, en prenant la traduction dans un sens très large, certains films sont meilleurs que les romans d'origine. Il peut y avoir une perte. pourquoi pas un gain?

Propos recueillis par ALEXIS NOUSS 